\title{
Feasibility of Expanding the Medication Abortion Provider Base in India to Include Ayurvedic Physicians and Nurses
}

\begin{abstract}
CONTEXT: The availability of trained abortion providers is limited in India. Allowing ayurvedic physicians and nurses to perform medication abortions may improve women's access to the procedure, but it is unclear whether these clinicians can provide these services safely and effectively.
\end{abstract}

\begin{abstract}
METHODS: Allopathic physicians, ayurvedic physicians and nurses (10 of each), none of whom had experience in abortion provision, were trained to perform medication abortions. In 2008-2010, these providers performed medication abortions in five clinics in Bihar and Jharkhand for 1,225 women with a pregnancy of up to eight weeks' gestation. A two-sided equivalence design was used to test whether providers' assessments of client eligibility and completeness of abortion matched those of an experienced physician "verifier," and whether medication abortions performed by nurses and ayurvedic physicians were as safe and effective as those done by allopathic physicians.
\end{abstract}

RESULTS: Failure rates were low (5-6\%), and those for nurses and ayurvedic physicians were statistically equivalent to those for allopathic physicians. Provider assessments of client eligibility and completeness of abortion differed from those of the verifier in only a small proportion of cases (3-4\% for eligibility and 4-5\% for completeness); these proportions, and rates of loss to follow-up, were statistically equivalent among provider types. No serious complications were observed, and services by all three groups of providers were acceptable to women.

\section{CONCLUSION: Findings support amending existing laws to improve women's access to medication abortion by expanding the provider base to include ayurvedic physicians and nurses.}

International Perspectives on Sexual and Reproductive Health, 2012, 38(3):133-142, doi: 10.1363/3813312

Abortion has been legal in India since the Medical Termination of Pregnancy Act went into effect on April 1, 1972; the use of mifepristone and misoprostol for medication abortion has been legally permitted since 2002. ${ }^{1,2}$ Women have the right to obtain abortions in a range of situations: if the mother's life, or her physical or mental health, is at risk; if the pregnancy is the result of rape; if the pregnancy is likely to result in the birth of an infant with physical or mental abnormalities; or if the pregnancy is the result of contraceptive failure. Abortions may be performed up to 20 weeks' gestation, and a woman undergoing an abortion does not require the consent of the husband or guardian if she is aged 18 or older. However, the procedures must be done in registered facilities, and only by gynecologists, or by other allopathic physicians who have undergone special training and obtained certification in the provision of surgical abortion. ${ }^{1}$ Over the years, the Government of India has made concerted efforts to increase access to safe abortion. For example, certification procedures have been rationalized, and rules and regulations amended, so that a certified provider now can perform a medication abortion in an unregistered facility, as long as he or she has access to a registered facility for backup. ${ }^{1}$

Despite this favorable legal scenario, access to safe abortion is limited in India; a large proportion of surgical abortions continue to take place outside of registered facilities, and a considerable proportion of both surgical and medication abortions are performed by uncertified providers. As a result, $8 \%$ of maternal deaths are attributable to unsafe abortion. ${ }^{3}$ The obstacles to access are wide ranging. Many women are unaware that abortion is legally available, ${ }^{4,5}$ or opt for an unsafe provider over a trained one because of concerns about confidentiality, cost and quality. ${ }^{6}$ Health system-related obstacles are also evident. A leading barrier to safe abortion has been lack of access to appropriate facilities and the limited availability of trained providers, ${ }^{7}$ nationally, only 12,510 facilities are registered to provide legal abortions, ${ }^{8}$ and few primary health centers have abortion facilities. ${ }^{9}$

Many developing countries, including Cambodia, Ethiopia, Nepal and Vietnam, allow trained nonphysicians (typically, nurse-midwives and physician assistants) to perform abortions. ${ }^{10}$ Evidence suggests that nonphysicians can provide first-trimester manual vacuum aspiration as safely as doctors can. ${ }^{11,12}$ Moreover, a recent study from Nepal, where nurses are legally permitted to terminate pregnancies using medication, found that medication abortions provided by nurses and auxiliary nurse-midwives were as successful as those provided by physicians. ${ }^{13}$

India has a pluralistic health sector that comprises a wide array of health providers; although allopathic (modern) medicine is the mainstay of the health system, indig-

\author{
By Shireen J. \\ Jejeebhoy, \\ Shveta Kalyanwala, \\ Shuchita Mundle, \\ Jaydeep Tank, \\ A.J. Francis Zavier, \\ Rajesh Kumar, \\ Rajib Acharya \\ and Nita Jha
}

Shireen J. Jejeebhoy is senior associate, A.J. Francis Zavier is program officer, the late Rajesh Kumar was assistant program officer and Rajib Acharya is associateall at the Population Council, New Delhi. Shveta Kalyanwala was senior program officer at the Population Council, New Delhi, at the time of the study. Shuchita Mundle is associate professor, Government Medical College, Nagpur, India. Jaydeep Tank is consultant, Ashwini Maternity and Surgical Hospital, Mumbai. Nita Jha was General Manager, Janani, Patna, India, at the time of the study. 
enous systems of medicine, including ayurveda, are also available. ${ }^{14}$ Ayurveda is a traditional system that relies to a large extent on plant materials, minerals and other naturally occurring substances, and is based on texts dating from as far back as 1500-1000 BC. Interest in formalizing this system of medicine increased following India's independence in 1947, and formal institutional training and certification in ayurveda became available at the university level in 1971 with the establishment of the Central Council for Indian Medicine. The ayurveda training program awards a Bachelor of Ayurvedic Medicine and Surgery degree, and teaches traditional concepts from the ayurvedic system of medicine as well as concepts from the allopathic system of medicine. ${ }^{14-16}$

As of January 2010, a total of 816,629 allopathic physicians were registered in India, including more than 27,000 obstetrician-gynecologists; in addition, there were 468,260 ayurvedic physicians and (as of December 2009) $1,073,638$ nurses. ${ }^{17,18}$ As mentioned earlier, only gynecologists and other allopathic physicians who have undergone training and received certification are legally permitted to provide abortions. Evidence from India on the feasibility of abortion provision by other competent providers is sparse. A recent study found that manual vacuum aspiration performed by nurses is as safe and effective as that done by allopathic physicians, ${ }^{12}$ however, similar studies related to medication abortion have not been conducted. In the current study, we sought to generate evidence that could inform policymakers and the medical community in India about the feasibility of expanding the categories of health care providers authorized to provide medication abortion. The study compared medication abortions performed by allopathic physicians with those provided by ayurvedic physicians or by nurses. Using an equivalence study design, we explored whether rates of complications and failed abortion resulting from medication abortions performed by nurses and ayurvedic physicians are equivalent to those of procedures done by recently trained allopathic physicians. A secondary objective was to explore differences by provider type in clinicians' ability to assess women's eligibility for medication abortion and the completeness of their abortions.

\section{METHODS}

\section{Setting}

The study was conducted in Bihar and Jharkhand, two poorly developed Indian states in which access to health services is limited. Together, these states have 120 million residents (accounting for 11\% of India's population); compared with India as a whole, they have considerably higher levels of poverty ( $43 \%$ vs. $26 \%$ of the population lives below the poverty line), ${ }^{19}$ maternal mortality (261 vs. 212 deaths per 100,000 births $)^{20}$ and unmet need for contraception among married women (23\% versus $13 \%) .{ }^{21}$ As of 2010, a total of 40,314 allopathic physicians were registered in the two states (33 per 100,000 population); ${ }^{17}$ about three-quarters of them were in the private sector. ${ }^{22}$ In ad- dition, there were 157,935 registered ayurvedic physicians (130 per 100,000 $)^{17}$ and about 22,000 registered nursing staff (18 per 100,000), including 10,881 nurses holding a diploma or degree. Facilities in these states are limited: The public sector has 2,217 allopathic hospitals, including community health centers ( 1.8 per 100,000 population), as well as 12 ayurvedic hospitals and 433 ayurvedic dispensaries (together, 0.4 per 100,000). ${ }^{17}$ Abortion services are even more limited: Just 51 obstetrician-gynecologists work in community health centers, ${ }^{23}$ and the two states have only 146 of the country's 12,510 registered abortion facilities $^{7}(0.1$ per 100,000 population, compared with 1.0 per 100,000 for India overall). These figures underscore the considerable difficulty that women in these states face when trying to obtain safe abortions. ${ }^{6,24}$

In India, individuals who have completed a higher secondary education (Class 12) may initiate training for a basic allopathic medical degree (MBBS), a Bachelor of Ayurvedic Medicine and Surgery degree (BAMS), or a diploma or BSc degree in nursing. An MBBS degree, which requires a 5.5-year course of study that includes basic obstetrics and gynecology, qualifies graduates to serve as general practitioners. The medical training for ayurvedic physicians also consists of a 5.5-year course of study and includes modules on obstetrics and gynecology. Under the National Rural Health Mission, ${ }^{*}$ ayurvedic physicians increasingly are being absorbed into the national health care system, ${ }^{25}$ where, in some states, they may be deployed as medical officers in charge of primary health centers and are expected to assist in such activities as performing deliveries and inserting IUDs. Nurses undergo a three-year (diploma) or four-year (BSc degree) program, and their curriculum also includes modules on gynecology and obstetrics. As a result of the considerable task shifting that has occurred under the National Rural Health Mission, nurses who complete additional training are permitted to perform deliveries and pelvic examinations where necessary and to insert IUDs independently. ${ }^{26,27}$ In many primary health centers, nurses are the mainstay of the reproductive and child health program. ${ }^{28}$ Although they are trained in aspects of obstetrics and gynecology, nurses and ayurvedic physicians are never involved in assessing abortion patients, determining gestational age, evaluating the completeness of abortions or prescribing drugs for medication abortion; however, some may assist the provider by counseling women, administering mifepristone or misoprostol or observing women until discharge.

The current study was conducted in five clinics operated by Janani, a nongovernmental organization affiliated with DKT International that provides a range of reproduc-

*The National Rural Health Mission was launched by the Government of India in 2005 to improve health care for the country's rural population, with a special focus on 18 states where public health status is below average and infrastructure is weak. One of the Mission's goals is to revitalize local health traditions and incorporate mainstream Indian systems of medicine into the public health system (source: National Rural Health Mission, Mission Document, 2005, <http://mohfw.nic.in/NRHM/ Documents/Mission_Document.pdf>, accessed Aug. 7, 2012). 
tive health services (including provision of contraceptives, HIV counseling, and first- and second-trimester abortion) through a network of facilities and outreach activities. Janani is a major provider of abortion services, and each clinic has at least one doctor who is certified to perform the procedure; the organization provides a large proportion of the abortions reported in Bihar and Jharkhand. Janani's charges are modest for first-trimester medication abortion or manual vacuum aspiration (399-499 rupees, or, at the time of the study, US\$9-\$11).

The five participating clinics are located in four urban areas, including Patna and Ranchi (the capitals of Bihar and Jharkhand, respectively), and serve largely low-income populations. The clinics were purposively selected on the basis of their high volume of clients and the geographical diversity of their settings.

\section{Design}

Four key issues informed our study design. First, to control for providers' level of experience in abortion provision, and to ensure that all three groups of providers started with the same level of experience, only providers who had never performed surgical or medication abortions, and had never (outside of their academic training) conducted pelvic examinations or assessed gestational age, were recruited for the study. Moreover, all underwent identical medication abortion training. The training, which was supervised by Ipas, lasted 10 days on average and included classroom lessons, practice sessions (using pelvic models), hands-on training at the facility and, finally, further training in the field, during which each provider was required to perform a minimum of 10 cases each of gestational age dating and assessment of the completion of medication abortion. We considered a training period of this length sufficient to ensure proficiency in gestational age dating and assessment of abortion completeness, as well as in counseling and other aspects of medication abortion provision; however, the duration was kept flexible, and if providers were experiencing difficulties the training was extended to permit more practice and build confidence.

Second, because ultrasound scanning is not necessary for the provision of early abortion, ${ }^{29}$ assessments of eligibility and completed abortion status were made solely on the basis of pelvic examinations. This protocol ensured that procedures were not overmedicalized (that is, did not rely on unnecessary preabortion procedures requiring sophisticated equipment ${ }^{29}$ ) and that the results of the study could be generalized to health facilities where ultrasound may not be available.

Third, since the providers in our study had not been certified to perform abortions (in the case of allopathic physicians) or were not legally permitted to do so (ayurvedic physicians and nurses), our study design included a "verifier" who confirmed the providers' evaluations and prescribed the medication for the abortions. The verifier was a certified abortion provider and typically had at least five years' experience in performing abortions.
Finally, because the staff at Janani clinics did not include uncertified allopathic physicians or ayurvedic physicians, or sufficient numbers of eligible nurses, most of the study providers were recruited from medical, nursing and ayurveda colleges or through advertisements placed in local newspapers; the only exceptions were six nurses who were Janani staff members.

Altogether, 30 providers participated in the study-10 allopathic physicians, 10 ayurvedic physicians and 10 nurses. All of them were female; although we did not impose any gender restrictions at the time of recruitment, it appears that most clinicians interested in providing abortion services are female, as few women opt for a male abortion provider in the conservative, gender-stratified social systems that prevail in the study areas. The median age of providers was identical in the three groups (28); however, the median number of months' experience that providers had as practicing clinicians varied from 12 among nurses and allopathic physicians to 23 among ayurvedic physicians.

Study providers took on the abortion-related care of all Janani clients who consented to join the study. Such care ranged from assessing eligibility and providing preabortion counseling to monitoring client status, performing follow-up and providing postabortion contraceptive counseling and support. The study was not a randomized controlled trial, as clients were not randomly assigned to a particular type of provider. Instead, one type of provider was assigned to each facility and remained until she had completed 35-40 medication abortions, which took approximately six weeks; all three types of providers were placed at each facility during the study. However, clients who came for an abortion did not know the type of clinician who would be providing services on the day of their visit, or the type who would succeed the current provider, as there was no particular sequence for the placement of physicians and nonphysicians. Moreover, the refusal rate was only $0.5 \%$, suggesting that few women opted for the clinic physician over the assigned study provider; moreover, the social and demographic profiles of clients served by the provider types were largely similar (Table 1). For these reasons, selection bias appears to have been limited, and the provider type assigned to each woman was, in effect, naturally random.

\begin{tabular}{|c|c|c|c|}
\hline Characteristic & $\begin{array}{l}\text { Ayurvedic } \\
\text { physicians } \\
(\mathrm{N}=461)\end{array}$ & $\begin{array}{l}\text { Nurses } \\
(\mathrm{N}=497)\end{array}$ & $\begin{array}{l}\text { Allopathic } \\
\text { physicians } \\
(\mathrm{N}=456)\end{array}$ \\
\hline Age (mean) & 26.8 & 26.6 & 27.1 \\
\hline$\geq 8$ yrs. of education (\%) & 80.9 & $73.4^{*}$ & 77.6 \\
\hline Never married (\%) & 5.2 & 3.8 & 4.2 \\
\hline No.of pregnancies (mean) & 3.4 & $3.7^{*}$ & 3.5 \\
\hline No.of previous abortions (mean) & 0.7 & 0.7 & 0.6 \\
\hline
\end{tabular}

${ }^{*} \mathrm{p}<.05$ for difference between nurses and ayurvedic physicians; differences for other comparisons were not statistically significant. 
Recruitment for the study took place from December 2008 to May 2010, ${ }^{*}$ although breaks in recruitment occurred at each clinic because a study provider was not always available. During the lengthy recruitment period, efforts were made to ensure that no changes occurred in key aspects of the service environment, including the routine services offered by the clinic, the adherence to the study protocol and the composition of the project staff (such as each facility's verifier and research coordinator). Costs of the abortion, as well as of any needed surgical procedures or treatment for complications, were borne by the project; no other compensation was provided to participants. Women were informed that the project would cover the costs of their procedure only after they had agreed to take part in the study, so that the offer of reimbursement of costs would not be perceived as unfair inducement to participate. The study was approved by the Population Council's institutional review board, the Indian Council of Medical Research's ethical review committee and Janani's ethical review board.

\section{Procedures}

At her initial visit, the client was introduced to the study provider, who counseled her about surgical and medication abortion options. The client was informed that she would be eligible for medication abortion if she had a uterine pregnancy of up to eight weeks' gestation (as confirmed by a urine pregnancy test and a pelvic examination) and had no contraindications for medication abortion. ${ }^{\dagger}$ In addition, she was informed that she would be required to return to the facility to take misoprostol (day 3) and assess the completeness of her abortion (day 15).

If the client opted for medication abortion, the provider explained the study protocol, described her medical background (i.e., allopathic physician, ayurvedic physician or nurse), informed the client that she was trained to provide medication abortion and obtained her consent to participate. The provider further explained that in order to participate, the client would need to have a hemoglobin level of at least $9 \mathrm{~g} / \mathrm{dL},{ }^{\dagger}$ undergo two pelvic examinations (by the study provider and verifier) and reside within an hour of the study site; moreover, she would be ineligible if, during the past week, she had tried to terminate the pregnancy using medication obtained from a chemist without a prescription. The criterion regarding proximity to the facility

\footnotetext{
*Recruitment took longer than anticipated because a large number of women did not meet the eligibility criteria, and because difficulties were encountered in identifying eligible providers who were willing to be placed in the selected facility for a short time.

†Contraindications were identified through history and routine examination, and included suspected ectopic pregnancy, hypertension, cardiovascular disease, allergy to mifepristone or misoprostol, previous cesarean section delivery, bronchial asthma and use of antitubercular drugs.

$\neq$ Government of India guidelines state that a hemoglobin level of $8 \mathrm{~g} / \mathrm{dL}$ or less is a contraindication for medication abortion. ${ }^{30,31}$

$\S$ Although women having medication abortions were not required by government guidelines to receive antibiotics, several institutions and facilities, including Janani, provide them as a rule; the study design conformed to the organization's protocol.
}

was included to enable speedy follow-up in case of complications and to comply with government guidelines stating that the client must reside near an appropriate health care facility ${ }^{30}$ the stipulation that the participant had not attempted to terminate the pregnancy in the past week was imposed to ensure that any complications that arose during the study would not be confounded with those resulting from inappropriate previous use of medication, as many women arrived at the Janani facility with such symptoms as uterine bleeding or incomplete abortion. If the woman met the aforementioned criteria, she provided a medical history (to rule out contraindications) and underwent two pelvic examinations (performed by the study provider and then the verifier using identical assessment criteria) to confirm her eligibility.

The study protocol entailed a minimum of three clinic visits. On the first day, women received $200 \mathrm{mg}$ mifepristone and were observed for 15 minutes. Following standard practice at the study sites, all women were given a three-day supply of antibiotics, as well as an analgesic (paracetamol) to be used at their discretion. They returned two days later, received $400 \mu \mathrm{g}$ of misoprostol and were observed for four to six hours. The dosages of mifepristone and misoprostol conformed to Government of India guidelines in place at the start of the trial. ${ }^{\$ 2,31}$ Misoprostol was administered orally, for two reasons. First, we wished to conform to government guidelines, ${ }^{31}$ which at the time of the trial advocated using the oral or vaginal route rather than sublingual or buccal administration. Second, practitioners and researchers on the study's technical advisory committee generally perceived that oral administration was more acceptable than vaginal administration to conservative Indian women. Women were informed that they would likely experience bleeding and pain, and were given the telephone numbers of the study provider and verifier in case they had side effects or concerns. On day 15 , women returned to the clinic and underwent another pelvic examination to assess the completeness of the abortion; an extended follow-up period was advised for women judged to have an incomplete abortion, who were asked to go home and return on day 21 to allow additional time for completion of the procedure. Given the high prevalence of anemia among Indian women in general, as well as concerns about further reductions in hemoglobin levels following medication abortion, hemoglobin was measured not only at enrollment but also on day 15 or (if extended follow-up was advised) on day 21.

The judgments of the verifier were considered the gold standard. In addition to confirming the study provider's assessments of the woman's eligibility to participate (day 1) and the completeness of her abortion (day 15 and, for those advised extended follow-up, day 21 as well), the verifier was responsible for prescribing the drugs for the medication abortion, providing backup support if required and performing manual vacuum aspiration on women with incomplete abortions or ongoing pregnancies. A research coordinator at each facility ensured that the provider and 
the verifier made independent assessments of women's eligibility and completed abortion status. For this purpose, the following procedure was adopted: Once the study provider had made her assessment, she left the consultation room and informed the research coordinator that she had completed her assessment; the research coordinator then called the verifier into the room to make her assessment.

In short, the study provider's responsibilities included obtaining the client's consent for medication abortion and for participation in the study; counseling the woman about abortion methods, the medication abortion procedure and its side effects, and postabortion contraception; ensuring that a pregnancy test and a hemoglobin test were completed; conducting the initial assessment for eligibility and for completed abortion status; providing the medication abortion drugs and observing the client as necessary; attending to client phone calls and unscheduled visits; referring clients with probable serious adverse events to the verifier; and providing postabortion contraception or referring women for postabortion tubal ligation or IUD insertion as appropriate. The verifier's responsibilities were primarily restricted to assessing eligibility and abortion completeness, and prescribing the medication abortion drugs; she also managed any serious adverse events, including performing manual vacuum aspiration for women with an incomplete abortion or an ongoing pregnancy.

The research coordinator collected social and demographic data from clients at enrollment, and information on clients' satisfaction and experiences during exit interviews on day 15 or 21 . The remainder of the study data were collected by the study provider (eligibility assessments on day 1 , abortion completeness assessments on days 15 and 21, information on mifepristone and misoprostol intake on days 1 and 3, and reports of any unscheduled contacts) and the verifier (assessments of eligibility, abortion completion and serious adverse events, if any).

\section{Outcome Indicators}

We examined several outcome measures. Our key indicator was the success of the abortion. As per Winikoff and colleagues, ${ }^{32}$ we defined any medication abortion that required subsequent surgical intervention (e.g., vacuum aspiration) for incomplete abortion or ongoing pregnancy* as a failure. Thus, the observed failure rate refers to the proportion of women for whom complete information was available who had an ongoing pregnancy on day 15 , or an incomplete abortion on day 15 or (if extended follow-up was advised) day 21. We also measured complication rates, defined as the percentage of women who experienced serious complications or required a blood transfusion or hospitalization.

In addition, because the study providers were inexperienced in conducting pelvic examinations, we calculated the proportion of clients whose eligibility had been incorrectly assessed by their provider (as compared with the verifier), including those who would have wrongly been denied entry into the study and those who would have wrongly been recruited. Similarly, we determined the proportion of study participants whose abortion completeness status was incorrectly assessed by the study provider. Loss to follow-up was defined as the proportion of study participants who took mifepristone on day 1 but did not return on day 3, 15 or 21. Finally, we measured acceptability, defined as the percentage of women reporting overall satisfaction with their experience.

\section{Statistical Analysis}

Data were analyzed using PASW Statistics 18.0 and Stata 10.1. We used a two-sided equivalence design to compare results among the three types of providers. An equivalence design is appropriate when the objective is to test whether one kind of intervention is indistinguishable from another, and whether differences observed between interventions are statistically insignificant. In this case, equivalence would be indicated if the $95 \%$ confidence intervals for the differences in outcomes (incorrect assessment of eligibility, completed abortion status and observed failure rates) between the allopathic physician group and each of the other two groups (ayurvedic physicians and nurses) fell within the margin of equivalence (extent of acceptable difference), which in our study was set to $\pm 5.5 \% .^{\dagger}$ This margin of equivalence was selected on the basis of clinical criteria as well as cost and feasibility.

To calculate the minimum sample size needed, we assumed a failure rate of $5 \%$ for all three types of providers on the basis of typical success rates reported by the World Health Organization (95-98\%) ${ }^{28}$ and by studies conducted in India that included oral administration of misoprostol (92-98\%). ${ }^{33,34}$ Given a two-sided equivalence with a margin of $5.5 \%, 80 \%$ power and a $95 \%$ confidence interval, we estimated that at least 330 cases needed to be assigned to each type of provider. We inflated the sample size by $10 \%$ to adjust for possible clustering and by an additional 5\% to account for loss to follow-up, yielding a required sample size of 1,140 women, or 380 per provider arm.

\section{RESULTS}

A total of 3,010 women presented for medication abortion; of these, more than half $(1,596)$ were ineligible for inclusion-15 refused to participate, 939 had attempted abortion on their own in the previous week, 556 resided more than one hour away from the facility, and 86 had contraindications (Web Appendix Figure 1). The remaining 1,414 women were screened for eligibility by a provider and verifier. On average, the women were aged 27 and had had between three and four pregnancies (including the current

\footnotetext{
*A complete abortion was defined as complete expulsion of products of conception, while an incomplete abortion was the retention of fetal tissue in the uterus, resulting in the need for uterine evacuation; both were determined clinically. A pregnancy was considered ongoing and viable if ultrasound revealed a fetal heartbeat.

tMore formally, in an equivalence trial, the margin of equivalence is the degree of inferiority (or superiority) of the test treatments to the control that the trial will attempt to exclude statistically (source: $\mathrm{Ng}$ et al., Choice of delta in equivalence testing, Drug Information Journal, 2001, 35(4):1517-1527).
} 
one); about three-quarters (73-81\%) had completed eight or more years of education (Table 1). Of screened women, 1,225 ( $87 \%$ ) were recruited into the study ( 404 by ayurvedic physicians, 416 by nurses and 405 by allopathic physicians); their social and demographic profile was virtually identical to that of those not recruited (not shown).

In 53 cases (3-4\%), the study provider's assessment of client eligibility for medication abortion differed from that of the verifier (Table 2). Nineteen women (1-2\%) would have been wrongly denied access by study providers (false negatives), and 34 women (2-3\%) would have been wrongly enrolled (false positives). However, sensitivity and specificity rates were high among all three groups of providers (98-99\% and 71-88\%, respectively), suggesting that convergence between assessments made by each provider group and the verifier were by and large high. Moreover, differences among provider types in the proportion of providers whose assessments differed from that of the verifier were negligible, and the confidence intervals of the differences fell within the predetermined margin of statistical equivalence. Furthermore, of the 53 cases in which the study provider's assessment of eligibility differed from that of the verifier, almost all (48) resulted from disparities in gestational age dating, regardless of provider type. In cases where the provider misclassified a woman as eligible, the difference in gestational age dating was generally narrow; indeed, in most instances, the verifier had estimated the gestational age to be nine weeks (i.e., only a week beyond the limit for eligibility).

Assessments of abortion completeness were conducted on day 15 for all 1,164 women who returned for followup, and again on day 21 for the 116 who were advised to return for an extended follow-up; therefore, 1,280 assessments of abortion completeness were conducted in total. The assessment made by the provider differed from that of the verifier in $4-5 \%$ of cases (Table 2). In 2-3\% of cases, providers wrongly classified the woman as having an incomplete abortion or continuing pregnancy (false negative); in 2\%, they wrongly assessed the case as a complete abortion (false positive). Sensitivity and specificity rates were high in all three groups of providers (97-98\% and $82-84 \%$, respectively), again indicating convergence between assessments made by study providers and those made by verifiers. The study provider and the verifier differed in 56 assessments (made on either day 15 or day 21); in 28 , the study provider had misreported the abortion as incomplete or concluded that the pregnancy was ongoing, while in the remaining 28 cases the study provider had misreported the abortion as complete (not shown). As with the eligibility assessments, differences among the three provider types in the proportion of providers whose assessment of abortion status differed from that of the verifier were negligible, and the confidence intervals for the differences between the verifier and each type of provider fell within the predetermined margin of statistical equivalence (Table 2)

We classified failure of medication abortions according to established methods. ${ }^{32}$ Of the 1,164 women who complied with the protocol and whose abortion completion status was assessed on day 15 or day $21,95 \%$ had successful medication abortions (not shown). Observed failure rates were similar for all provider types (5-6\%); for each type, $2 \%$ of women had an ongoing pregnancy and $3 \%$ had an incomplete abortion (Table 3). The confidence intervals of the differences in failure rates between allopathic physicians and ayurvedic physicians, and between allopathic physicians and nurses, fell within the predetermined margin of statistical equivalence, implying that the failure rates observed for ayurvedic physicians and nurses were statistically equivalent to those observed for allopath-

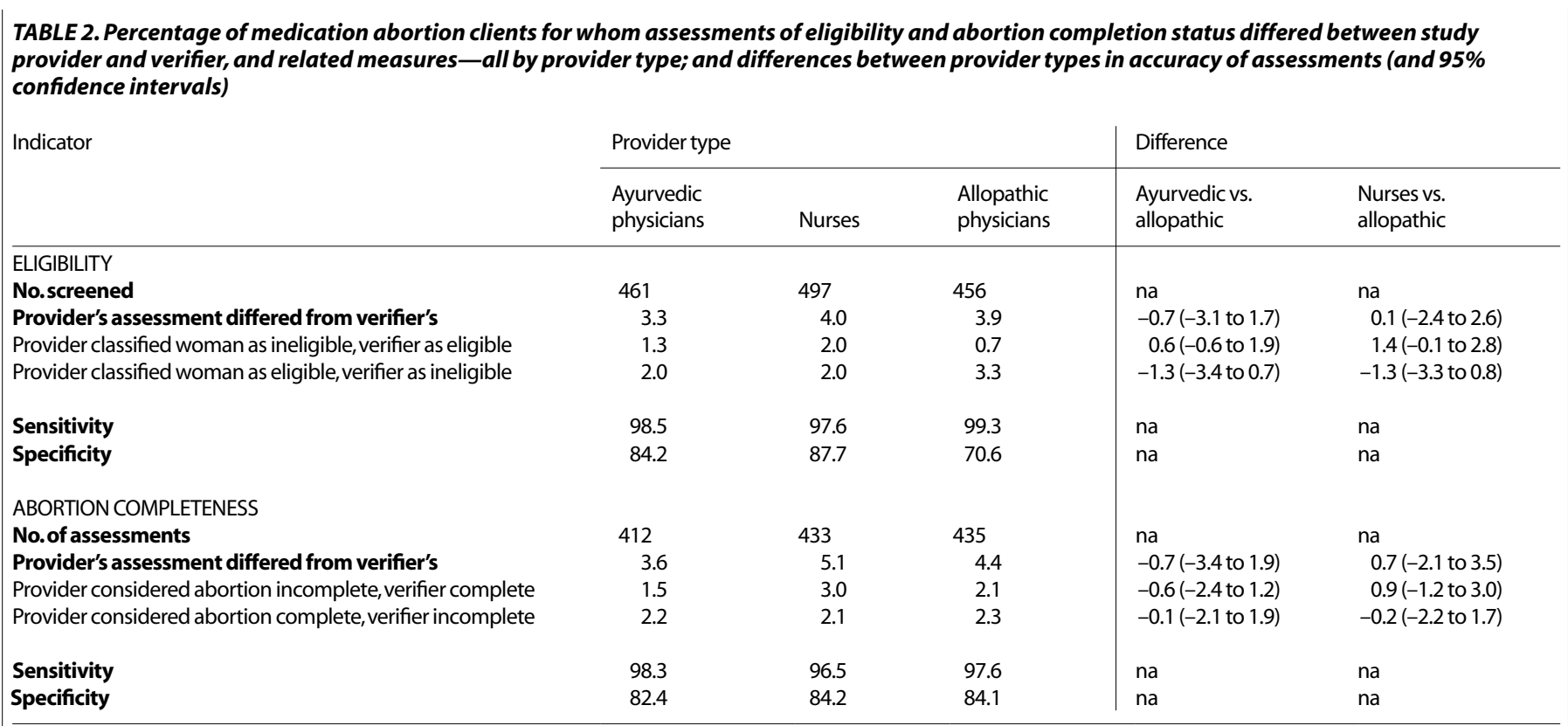

Notes: Equivalence is indicated when the $95 \%$ confidence interval for the difference in failure rates between the allopathic physician group and each of the other two groups (ayurvedic physicians and nurses) lies within the predetermined margin of equivalence $( \pm 5.5 \%)$. na $=$ not applicable. 
TABLE 3. Medication abortion failure rates, by provider type, and differences in failure rates between provider types (and 95\% confidence intervals)

\begin{tabular}{llll|lr} 
Indicator & Provider type & & Difference \\
\cline { 2 - 6 } & $\begin{array}{l}\text { Ayurvedic physicians } \\
(\mathrm{N}=382)\end{array}$ & $\begin{array}{l}\text { Nurses } \\
(\mathrm{N}=393)\end{array}$ & $\begin{array}{l}\text { Allopathic physicians } \\
(\mathrm{N}=389)\end{array}$ & Ayurvedic vs. allopathic Nurses vs. allopathic \\
\hline Observed failure rate & 5.5 & 4.6 & 4.6 & $0.9(-2.2$ to 4.0$)$ & $-0.1(-3.0$ to 2.9$)$ \\
Ongoing pregnancy & 2.1 & 1.8 & 1.8 & $0.3(-1.7$ to 2.3) \\
Incomplete abortion & 3.4 & 2.8 & 2.8 & $0.6(-1.9$ to 3.0) & $0.0(-1.9$ to 1.8$)$ \\
\hline
\end{tabular}

Note: Equivalence is indicated when the $95 \%$ confidence interval for the difference in failure rates between the allopathic physician group and each of the

other two groups (ayurvedic physicians and nurses) lie within the predetermined margin of equivalence $( \pm 5.5 \%)$.

ic physicians. All women with an ongoing pregnancy or incomplete abortion underwent MVA.

The proportion of women lost to follow-up was small: Of the 1,225 women enrolled in the study, just 5\% failed to return on days 3, 15 or 21 (not shown). Proportions for the three provider types ranged from $4 \%$ to $6 \%$. Overall, just $0.2-2 \%$ of recruited women did not return on day 3 for misoprostol, and $2-5 \%$ did not return on day 15 or 21 for the follow-up visit. The confidence intervals for the differences in loss to follow-up fell within the predetermined margin of statistical equivalence for each provider type, both for day 3 and for day 15 or 21 .

Relatively small proportions of women made phone calls (5\%) or unscheduled visits (4\%) during the study, and most of these related to anxiety or uncertainty about the procedure or concerns about the extent and duration of their bleeding (not shown). No women had serious complications, or required a blood transfusion or hospitalization. One-fifth of women had a decline in hemoglobin levels of more than $1.0 \mathrm{~g} / \mathrm{dL}$; fewer than $1 \%$ in each group had a hemoglobin level of less than $8.0 \mathrm{~g} / \mathrm{dL}$ at follow-up. Hemoglobin levels did not differ by type of provider.

Finally, exit interviews showed that regardless of provider type, women were overwhelmingly satisfied with the services they received (98-99\%; Table 4). In all three groups, nearly all clients indicated that the provider had given them enough time to discuss their situation (100\%), had explained the procedure clearly (98-100\%) and had counseled them about postabortion contraception (98$100 \%$ ). Moreover, almost all women whose procedure had been done by an ayurvedic physician or a nurse reported being willing to undergo medication abortion again from the same type of provider should the need arise. The vast majority of clients reported that they found the bleeding and pain from the procedure tolerable (88-91\% and 90$96 \%$, respectively).

\section{DISCUSSION}

The results showed that according to our definition of equivalence, ayurvedic physicians and nurses can provide medication abortion with the same degree of safety and effectiveness as allopathic physicians. Indeed, the high rates of sensitivity and specificity suggest that all three types of providers assessed eligibility and completeness of abortion as well as the verifier did. Furthermore, observed failure rates were similar to rates obtained in previous studies ${ }^{33-35}$ in which misoprostol was administered orally, although they were somewhat higher than rates obtained in studies using vaginal or sublingual administration. ${ }^{34,35}$ Loss to follow-up was similar for all three types of providers, and was comparable to levels in previous studies conducted among experienced physicians. ${ }^{33,36}$ Rates of client satisfaction and willingness to undergo abortion from a nurse or ayurvedic physician were very high.

Study providers' low observed failure rates and high levels of accuracy in assessing gestational age and completion of abortion are particularly impressive given that these outcomes were obtained in the absence of ultrasound by providers who had never previously performed abortions or pelvic examinations. Given the association between experience and skill, a longer training period or the inclusion of more experienced providers likely would have resulted in even better outcomes.

We acknowledge several limitations of the study. The most obvious is that the study was not randomized. Providers were placed sequentially, rather than simultaneously, in each facility, resulting in pseudorandomization of clients; however, the similarities in social and demographic characteristics of each group's clients suggest that the study design did not result in substantially different groups of women being served by each provider type. Moreover, the proportion of women who refused to participate in the study (and opted instead to obtain an abortion from the regular clinic provider) was minimal for all types of providers, suggesting that the extent of any bias was limited.

We also acknowledge the potential bias introduced by the presence of the verifiers, whose task it was to override

TABLE 4. Percentage of medication abortion clients who reported satisfaction with selected aspects of the procedure, by provider type

\begin{tabular}{lcrc} 
Outcome & $\begin{array}{c}\text { Ayurvedic } \\
\text { physicians } \\
(\mathrm{N}=382)\end{array}$ & $\begin{array}{c}\text { Nurses } \\
(\mathrm{N}=393)\end{array}$ & $\begin{array}{c}\text { Allopathic } \\
\text { physicians } \\
(\mathrm{N}=389)\end{array}$ \\
\hline Satisfaction & & & \\
Satisfied with overall services & 97.6 & 99.0 & 98.7 \\
Provider spent enough time explaining situation & 100.0 & 100.0 & 100.0 \\
Provider explained procedure clearly & 99.2 & 98.0 & 99.7 \\
Provider counseled about postabortion contraception & 99.5 & 97.5 & 100.0 \\
Willing to have future abortion with same provider type & 99.7 & 99.7 & 100.0 \\
& & & \\
Acceptability of side effects & & & \\
Bleeding was tolerable & 88.2 & 90.6 & 90.7 \\
Pain was tolerable & 95.8 & 90.1 & 95.1 \\
\hline Note: Analysis restricted to women for whom complete information was available. &
\end{tabular}


any incorrect assessments of eligibility or abortion completeness. However, the verifiers' involvement most likely did not affect the results in any major way, because the numbers of women excluded from or included in the study because of verifier intervention were small.

Another potential limitation is the relevance of the findings to a public-sector environment. Our study was conducted in the facilities of a nongovernmental service provider whose quality standards may be quite different from those of a typical public-sector facility, which raises questions about the applicability of our findings to other settings. Although this is a concern, the National Rural Health Mission has increased its focus on quality assurance (including an emphasis on counseling) and its interest in task shifting and task sharing, and it has proposed including mifepristone and misoprostol in the schedule of drugs available in public-sector facilities; these steps suggest growing attention to quality of care and provision of medication abortion in public facilities. At the same time, we reiterate that the study procedures followed in every way the guidelines adopted for public-sector provision of medication abortion. For these reasons, we believe that the conditions in our study are, with appropriate attention to quality and counseling, replicable in public-sector facilities.

Finally, the fact that the study population was restricted to women residing within an hour of the facility, allowing for easy access to care in case of complications (and complying with government regulations), raises questions about the applicability of the findings to situations in which the distance to the facility is longer. However, given the growing availability of emergency ambulance services and facilities that provide medication abortion, a typical rural woman will likely be able to reach an appropriate facility in a timely way in the rare likelihood of a serious adverse event.

Lessons for replication may be drawn from our study procedures. For example, the training curriculum, designed and supervised by Ipas, was adapted from that organization's extensive experience providing abortionrelated training to medical officers in the public sector. In the trainers' experience, a 10-day program (with provision for extending the duration of training for clinicians requiring additional hands-on experience) was sufficient to hone providers' skills in assessing gestational age and completeness of abortion, as well as in counseling. Although a longer training period undoubtedly would have given providers more experience and confidence, the low observed failure rates in all three provider groups indicate that a 10-day program with built-in flexibility provides a sufficient duration of training.

The findings have considerable relevance to the recent activities of the National Rural Health Mission. As noted earlier, the Mission is increasingly committed to task shifting and task sharing; already, ayurvedic physicians have been placed in a number of primary health centers, and nurses' responsibilities in such endeavors as skilled birth attendance have significantly expanded. Efforts are under way, moreover, to ensure that abortion services, with appropriate backup in case of complications, are available at the primary health center level. Notwithstanding these limitations, our findings are encouraging and make a strong case in support of the National Rural Health Mission's activities, and of further efforts to amend laws to expand the medication abortion provider base and thereby increase women's access to safe abortion in India.

\section{REFERENCES}

1. Government of India, The Medical Termination of Pregnancy Rules (Amendment), New Delhi: Government of India, 2003.

2. Drugs Controller General, Number of Permission and Date of Issue MF-7059/06, New Delhi: Government of India, 2006.

3. Registrar General, Maternal Mortality in India, 1997-2003: Trends, Causes and Risk Factors, New Delhi: Registrar General, India, 2006.

4. Jejeebhoy SJ et al., Increasing Access to Safe Abortion in Rural Maharashtra: Outcomes of a Comprehensive Abortion Care Model, New Delhi: Population Council, 2011

5. Jejeebhoy SJ et al., Increasing Access to Safe Abortion in Rural Rajasthan: Outcomes of a Comprehensive Abortion Care Model, New Delhi: Population Council, 2011

6. Barua A and Apte H, Quality of abortion care: perspectives of clients and providers in Jharkhand, Economic and Political Weekly, 2007, 42(48):71-80.

7. Ministry of Health and Family Welfare (MOHFW), Family Welfare Statistics in India, New Delhi: MOHFW, 2009.

8. Jejeebhoy SJ et al., Assessing Abortion-Related Experiences and Needs in Four Districts of Maharashtra and Rajasthan, New Delhi: Population Council, 2010

9. Santhya KG and Verma S, Induced abortion, in: Jejeebhoy S, ed. Looking Back, Looking Forward: A Profile of Sexual and Reproductive Health in India, Jaipur, India: Rawat Publications, 2004, pp. 88-104.

10. Berer M, Provision of abortion by mid-level providers: international policy, practice and perspectives, Bulletin of the World Health Organization, 2009, 87(1):58-63.

11. Warriner IK et al., Rates of complication in first-trimester manual vacuum aspiration abortion done by doctors and mid-level providers in South Africa and Vietnam: a randomised controlled equivalence trial, Lancet, 2006, 368(9551):1965-1972.

12. Jejeebhoy $\mathrm{SJ}$ et al., Can nurses perform manual vacuum aspiration (MVA) as safely and effectively as physicians? Evidence from India, Contraception, 2011, 84(6):615-621.

13. Warriner IK et al., Can midlevel health-care providers administer early medical abortion as safely and effectively as doctors? A randomised controlled equivalence trial in Nepal, Lancet, 2011, 377(9772):1155-1161.

14. World Health Organization (WHO), Not Enough Here ... Too Many There ... Health Workforce in India, New Delhi: WHO, 2007.

15. Central Council for Research in Ayurvedic Sciences, MOHFW, Ayurveda: origin, <http://www.ccras.nic.in/ayurveda/ 20081010_origin.htm>, accessed Jan. 13, 2012.

16. Bhadalikar DS, Perspective of ayurved in India, paper presented at the Regional Consultation on Expanding Access to Safe Abortion and Post-Abortion Care, New Delhi, Mar. 7-8, 2011.

17. Central Bureau of Health Intelligence, National Health Profile (NHP) of India-2010, New Delhi: Government of India, 2010.

18. Federation of Obstetric and Gynecological Societies of India, FOGSI profile, <http://www.fogsi.org/index.php?option=com content\&view=article\&id=3\&Itemid=23>, accessed Jul. 24, 2012

19. Planning Commission, India, Poverty estimates for 2004-2005, 2007, <http://planningcommission.gov.in/news/prmar07.pdf>, accessed Oct. 8, 2009. 
20. Office of the Registrar General, Special Bulletin on Maternal Mortality in India 2007-09: Sample Registration System, New Delhi: Office of the Registrar General, India, 2011.

21. International Institute for Population Sciences (IIPS) and Macro International, National Family Health Survey (NFHS-3), 2005-06: India, Vol. 1, Mumbai: IIPS, 2007.

22. Rao M et al., Human resources for health in India, Lancet, 2011, 377(9765):587-598.

23. MOHFW, Health profile of states, <http://www.mohfw.nic.in/ NRHM/Health_Profile.htm>, accessed Jan. 11, 2011.

24. Barnes L, Abortion options for rural women: case studies from the villages of Bokaro district, Jharkhand, working paper, Mumbai: Centre for Enquiry into Health and Allied Themes/Healthwatch, 2003.

25. MOHFW, National Rural Health Mission: Meeting People's Health Needs: Framework for Implementation 2005-2012, New Delhi: MOHFW, 2005.

26. MOHFW, Indian Public Health Standards (IPHS) For Sub-Centres: Guidelines, New Delhi: Government of India, 2006.

27. Department of Health, Government of India, The Drugs and Cosmetics Act and Rules, 2003, <http://cdsco.nic.in/html/ copy\%20of\%201.\%20d\&cact121.pdf>, accessed Jan. 13, 2012.

28. IIPS, India Facility Survey (Under Reproductive and Child Health Project) Phase II, 2003, IIPS: Mumbai, 2005.

29. WHO, Safe Abortion: Technical and Policy Guidance for Health Systems, Geneva: WHO, 2003.

30. MOHFW, Comprehensive Abortion Care: Training and Service Delivery Guideline, New Delhi: Government of India, 2010.

31. WHO, Use of RU-486 with Misoprostol for Early Abortion in IndiaGuidelines for Medical Officers, New Delhi: All India Institute of Medical Sciences, 2003

32. Winikoff B, Ellertson C and Clark S, Analysis of failure in medical abortion, Contraception, 1996, 54(6):323-327.

33. Coyaji K et al., Mifepristone-misoprostol abortion: a trial in rural and urban Maharashtra, India, Contraception, 2002, 66(1):33-40.

34. von Hertzen $\mathrm{H}$ et al., WHO multinational study of three misoprostol regimens after mifepristone for early medical abortion. I: Efficacy, BJOG, 2003, 110(9):808-818.

35. Mittal S et al., Comparison of oral versus vaginal misoprostol $\&$ continued use of misoprostol after mifepristone for early medical abortion, Indian Journal of Medical Research, 2005, 122(2):132-136.

36. Mundle $S$ et al., Increasing access to safe abortion services in rural India: experiences with medical abortion in a primary health center, Contraception, 2007, 76(1):66-70.

\section{RESUMEN}

Contexto: La disponibilidad de prestadores capacitados de servicios de aborto es limitada en India. El permitir que médicos ayurvedas y enfermeras realicen abortos con medicamentos puede mejorar el acceso de las mujeres al procedimiento, pero no es claro si este personal clínico puede prestar estos servicios de manera segura y efectiva.

Métodos: Médicos alópatas, médicos ayurvedas y enfermeras (10 de cada uno), ninguno de los cuales tenía experiencia en la prestación de aborto, fueron capacitados para realizar abortos con medicamentos. Entre 2008 y 2010, estos proveedores realizaron abortos con medicamentos en cinco clínicas ubicadas en los estados de Bihar y Jharkhand entre 1,225 mujeres con embarazos de hasta ocho semanas de gestación. Se usó un diseño de equivalencia para verificar si la valoración de la elegibilidad de las pacientes del aborto por parte de estos proveedores y su evaluación de lo incompleto que eran los abortos coincidian con las determinaciones de un médico experimentado "verificador". El análisis también consideró si los abortos con medicamentos realizados por las enfermeras y los médicos ayurvedas eran tan seguros y efectivos como los realizados por médicos alópatas.

Resultados: Las tasas de falla de los abortos con medicamentos fueron bajas (5-6\%) en general, y las tasas de falla de los abortos provistos por enfermeras y médicos ayurvedas fueron estadísticamente iguales a las tasas de los médicos alópatas. Las determinaciones de los proveedores en cuanto a la elegibilidad de las pacientes para el procedimiento y lo incompleto de los abortos difirieron con respecto a las determinaciones del "verificador" solo en una pequeña proporción de los casos (3-4\% para elegibilidad y 4-5\% para lo incompleto del procedimiento). Estas proporciones y las tasas de pérdida de seguimiento fueron estadísticamente iguales entre los tres tipos de proveedores. No se observaron complicaciones serias y los servicios de los tres grupos de proveedores fueron aceptables para las mujeres.

Conclusión: Los hallazgos apoyan la enmienda de las leyes existentes para mejorar el acceso de las mujeres al aborto con medicamentos mediante la expansión de los prestadores autorizados para prestar los servicios de aborto para incluir a médicos ayurvedas y enfermeras.

\section{RÉSUMÉ}

Contexte: L'accès à des prestataires formés à la pratique de l'avortement est limité en Inde. En autorisant les praticiens ayurvédiques et les infirmières à pratiquer l'avortement médicamenteux, on pourrait améliorer l'accès des femmes à la procédure, mais il n'est pas clairement établi que ces cliniciens puissent assurer ces services sans risques et de manière efficace. Méthodes: Des médecins allopathes, des praticiens de la médecine ayurvédique et des infirmières (10 dans chaque catégorie), sans expérience aucune de la pratique de l'avortement, ont été formés à celle de l'avortement médicamenteux. En 2008-2010, ces prestataires ont pratiqué cette forme d'IVG dans cinq cliniques du Bihar et du Jharkhand, au service de 1.225 femmes enceintes d'un maximum de huit semaines. Les évaluations des prestataires concernant l'admissibilité des clientes et l'accomplissement total de l'avortement par rapport à celles d'un médecin «vérificateur» expérimenté ont été testées selon un plan d'équivalence bilatéral, de même que l'absence de risques et que l'efficacité des procédures médicamenteuses pratiquées par les infirmières et les praticiens ayurvédiques par rapport à leurs confrères allopathes.

Résultats: Les taux d'échec se sont avérés faibles (5\% à $6 \%)$, avec équivalence statistique entre ceux relevés pour les infirmières et les praticiens ayurvédiques et ceux associés aux médecins allopathes. Les évaluations des prestataires quant à l'admissibilité des clientes et l'accomplissement total de l'avortement ne diffèrent de celles du vérificateur que dans une faible proportion des cas (3\% à 4\% pour l'admissibilité et 4\% à $5 \%$ pour l'accomplissement). Ces proportions, de même que les taux de perte au suivi, sont statistiquement équivalentes 
parmi les différents types de prestataires. Aucune complication grave n'a été observée et les services apportés par chacun des trois groupes de prestataires se sont avérés acceptables aux yeux des femmes.

Conclusion: Ces constatations appuient l'argument en faveur de l'amendement des lois existantes pour améliorer l'accès des femmes à l'avortement médicamenteux en étendant la base de prestataires aux praticiens ayurvédiques et aux infirmières.

\section{Acknowledgments}

The study was supported by the Swedish International Development Cooperation Agency and the David and Lucile Packard Foundation. The authors thank Sangeeta Batra, Manju Chhugani, Lester Coutinho, Kurus Coyaji, Bela Ganatra, Sharad Iyengar, Manisha Malhotra, Vinoj Manning, Suneeta Mittal, Malabika Roy and Iqbal Shah for their valuable comments on the study protocol or on drafts of this article; the Concept Foundation, for supplying the drugs used in the study; Janani, for housing the project; our study teams, for ensuring the smooth functioning of the project; Shilpi Rampal, for office assistance; and M.A. Jose for general administration.

Author contact: sjejeebhoy@popcouncil.org 


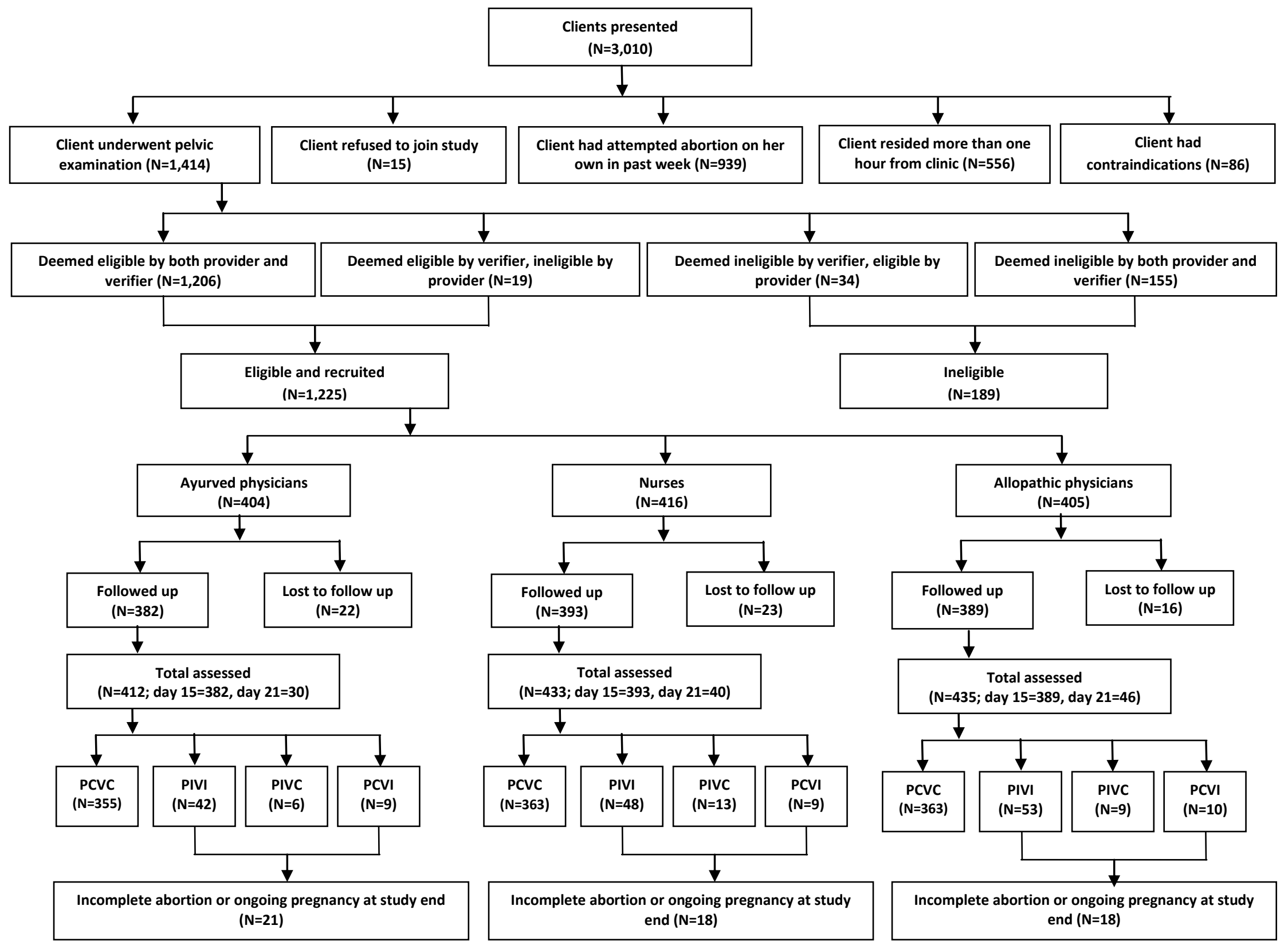

Notes: PCVC=Considered complete by both provider and verifier. PIVI=Considered incomplete abortion or ongoing pregnancy by both provider and verifier. PIVC=Considered complete by verifier, incomplete abortion or continuing pregnancy by provider. PCVI=Considered incomplete abortion or continuing pregnancy by verifier, complete abortion by provider. 